of Ipilimumab/Nivolumab and five cycles of Pembrolizumab, he developed bilateral anterior uveitis and raised intra-ocular pressures (IOPs). His ocular inflammation was treated with Maxidex, and IOPs controlled with Latanoprost and Betagan. Unfortunately he developed further irAEs, including grade 2 colitis, adrenal insufficiency and diabetes. His immunotherapy was discontinued due to cumulative toxicity despite complete radiological response. Interestingly, after the resolution of all ocular inflammation, poor vision in the left eye persisted (6/24) and electrophysiology led to a diagnosis of melanoma-associated retinopathy (MAR), a paraneoplastic autoimmune complication of melanoma (Fig. 2). This was confirmed by the presence of anti-recoverin and carbonic anhydrase II antibodies.

Clinicians must be vigilant for inflammatory adverse effects of immunotherapy in the eye. Often the effects are minor; however, rarely, certain cases can be sight threatening. None of our patients had immunotherapy discontinued due to ocular irAEs alone, and all were managed with topical and oral steroids. Our data highlight the higher-thananticipated incidence of ocular inflammation in patients on novel therapies for metastatic melanoma in Northern Ireland.

\section{Compliance with ethical standards}

Conflict of interest The authors declare that they have no conflict of interest.

Publisher's note: Springer Nature remains neutral with regard to jurisdictional claims in published maps and institutional affiliations.

\section{References}

1. Karimkhani C, Reddy BY, Dellavalle RP, et al. Novel therapies for unresectable and metastatic melanoma. BMJ. 2017;359:j5174.

2. Andrews S, Holden R. Characteristics and management of immune related adverse effects associated with Ipilimumab, a new immunotherapy for metastatic melanoma. Cancer Manag Res. 2012;4:299-307.

3. Hahn L, Pepple KL. Bilateral neuroretinitis and anterior uveitis following Ipilimumab treatment for metastatic melanoma. J Ophthalmic Inflamm Infect. 2016;6:14.

4. Hanna KS. A rare case of pembrolizumab-induced uveitis in a patient with metastatic melanoma. Pharmacotherapy. 2016;36:e183-8.

5. Sarny S, Neumayer M, Kofler J, El-Shabrawi Y. Ocular toxicity due to Trametinib and Dabrafenib. BMC Ophthalmol. 2017;17:146.

\title{
Self-tattooing of eyeball with inadvertent corneoscleral perforation: the implication of social media
}

\author{
Jia Yu Ng${ }^{1} \cdot$ Darren Shu Jeng Ting $\mathbb{D}^{1,2} \cdot$ Daniela Vaideanu-Collins ${ }^{1} \cdot$ Hardeep Singh Mudhar ${ }^{3} \cdot$ Bart Wagner $^{4} \cdot$ \\ Patricia Goggin $^{5}$. Andrew Gibson ${ }^{1} \cdot$ Trushar Patel $^{1}$
}

Received: 30 April 2019 / Accepted: 2 May 2019 / Published online: 5 June 2019

(c) The Royal College of Ophthalmologists 2019

These authors contributed equally: Jia Yu Ng, Darren Shu Jeng Ting

Darren Shu Jeng Ting

ting.darren@gmail.com

1 Department of Ophthalmology, The James Cook University Hospital, Middlesbrough, UK

2 Academic Ophthalmology, Division of Clinical Neuroscience, School of Medicine, University of Nottingham, Nottingham, UK

3 National Specialist Ophthalmic Pathology Service, Department of Histopathology, Royal Hallamshire Hospital, Sheffield, UK

4 Electron Microscopy Unit, Department of Histopathology, Royal Hallamshire Hospital, Sheffield, UK

5 Biomedical Imaging Unit, University Hospital Southampton NHS Trust, Southampton, UK
To the Editor

Body modification procedures are becoming increasingly popular, especially, in the social media generation where an easily accessible self-care or Do-It-Yourself (DIY) culture is common [1]. We report a case of inadvertent corneoscleral perforation following self-attempt of bilateral ocular tattooing guided by a YouTube video.

A 34-year-old Caucasian man presented to our eye casualty with an acute painful right eye after performing a DIY ocular tattooing procedure using a 31-gauge hypodermic needle, and Fibracolor white baby finger paint purchased online. After multiple prompting during the initial consultation, the patient disclosed a past history of bilateral laser epithelial keratomileusis (LASEK) for myopia, radical bilateral bulbar conjunctivalectomy for post-LASEK chronic bulbar conjunctival 


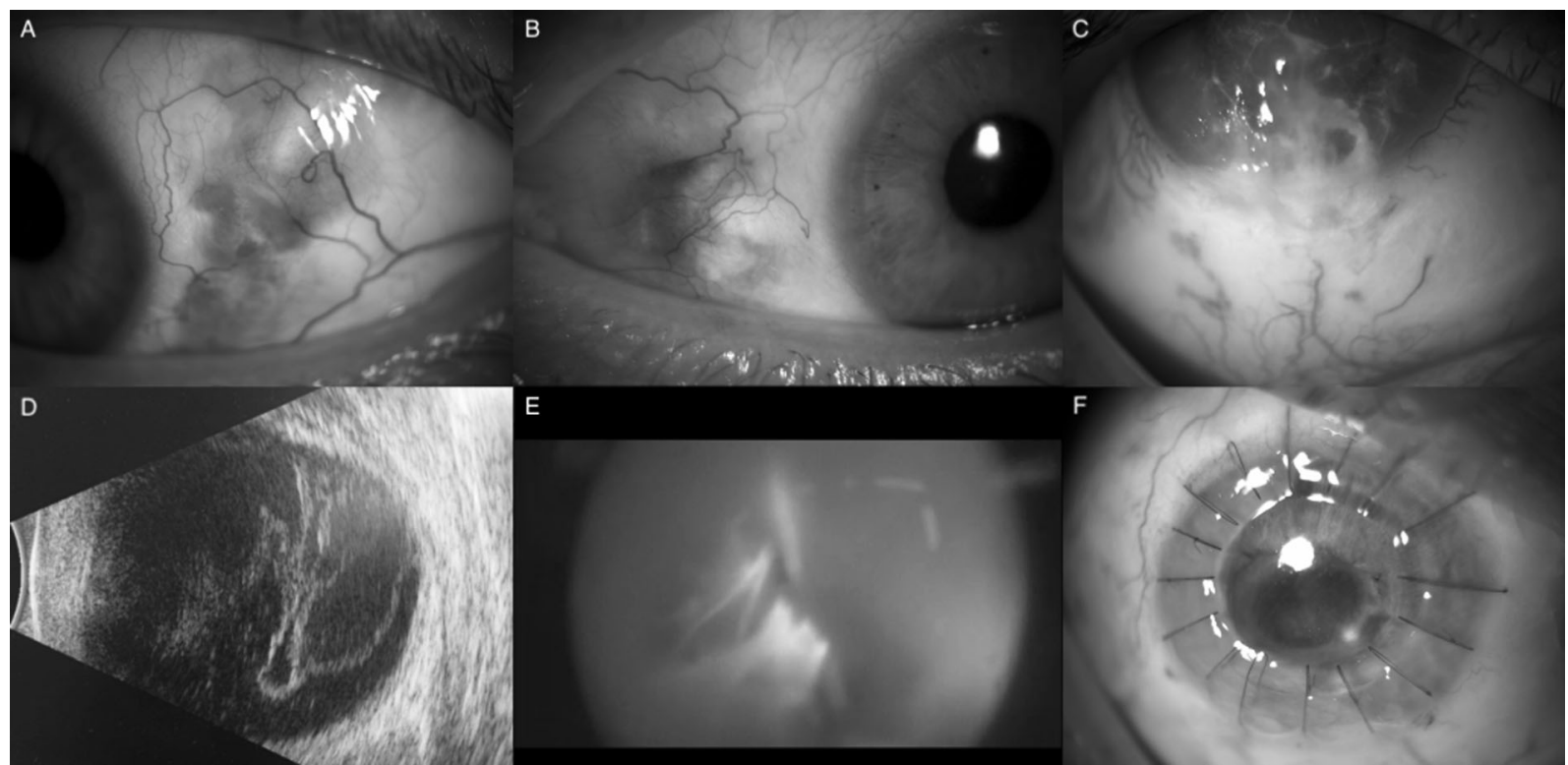

Fig. 1 a Slit-lamp photography of the right eye showing dark patches on the sclera related to scleromalacia. b Slit-lamp photography of the left eye showing white paint material in the sub-Tenon space and scleromalacia. c Slit-lamp photography of the right eye showing fibrinous material in the anterior chamber with hypermature white cataract obscuring the fundal view. d Ocular B-scan ultrasound of the right eye showed discrete, mobile echogenic particles within the vitreous cavity. e Extensive contamination of the right vitreous with white paint material noted intraoperatively. f Slit-lamp photography showing a clear fullthickness/penetrating corneal graft with marked transpupillary membrane
Fig. 2 a Hematoxylin and Eosin (H\&E) stained cell block preparation from the vitreous biopsy showing macrophages containing paint particles in their cytoplasm. This is clearly shown in the top right inset plate.

b CD68 immunohistochemistry, confirming that the cells containing the paint particles are macrophages. Brown is positive staining. c Transmission electron micrograph (TEM) showing the larger electron dense paint particles (black arrows) and the smaller more crystalline paint particles (white arrows). The white holes are artefacts of tissue processing. d Higher power TEM showing the larger, more electron dense paint particles just below of centre and the accompanying, smaller, more numerous, less electron dense, more crystalline paint particles. e The energy dispersive microanalysis of X-ray read-out showing the various elements present in the white paint: $\mathrm{C}$ carbon, $\mathrm{O}$ oxygen, $\mathrm{Al}$ aluminium, $\mathrm{Si}$ silicon, $\mathrm{P}$ phosphorus, Ca calcium, Ti titanium $\mathrm{Cu}$ copper
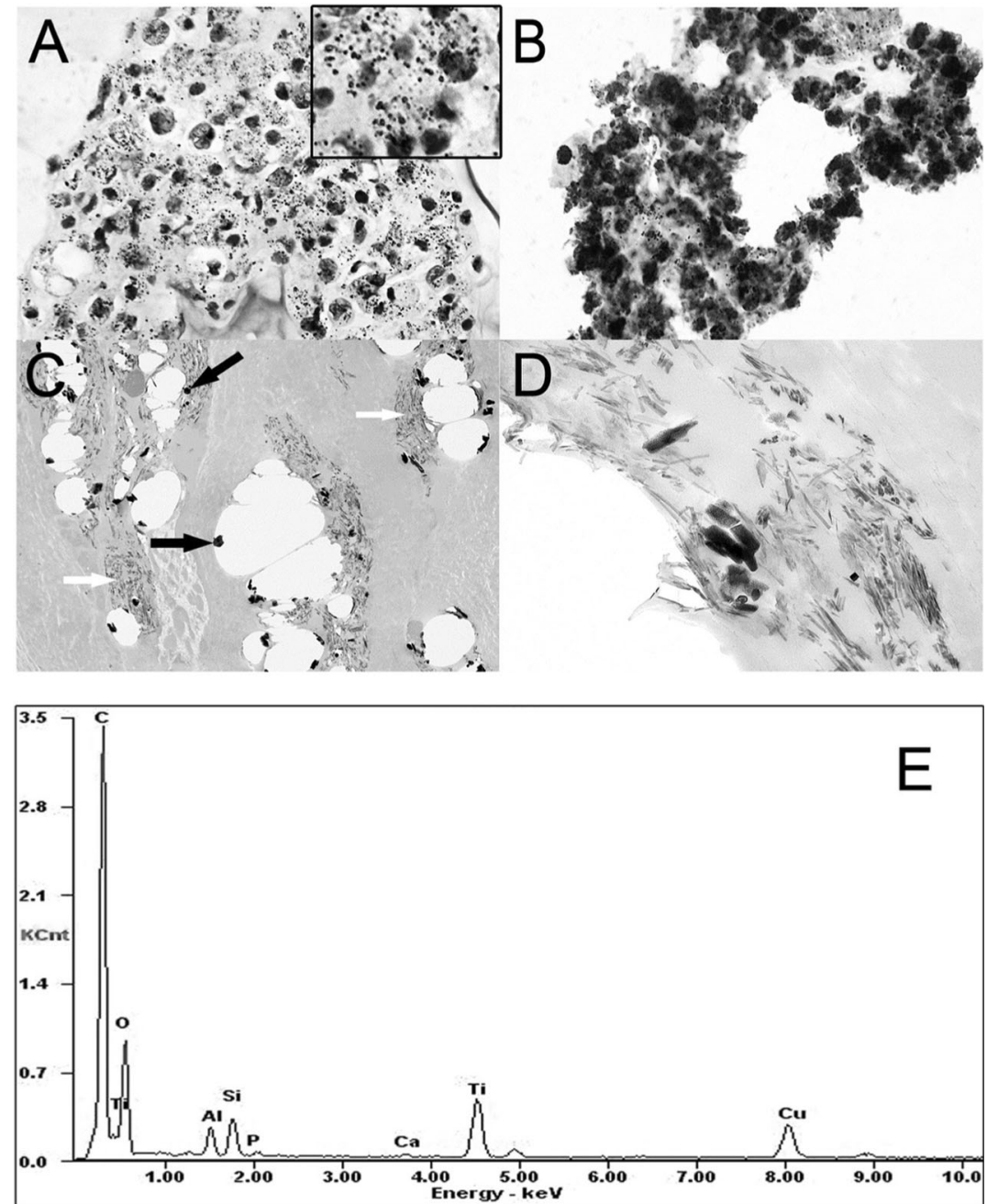
hyperaemia, consequent cosmetically unacceptable scleromalacia and self-ocular tattooing.

At presentation, the corrected-distance-visual-acuity was hand movement (right eye) and 6/5 (left eye). There were bilateral scleromalacia involving $360^{\circ}$ of the bulbar associated with overlying calcified plaques (Fig. 1a, b). Examination revealed a right flat anterior chamber with white pseudohypopyon (Fig. 1c) and an intraocular pressure (IOP) of $8 \mathrm{mmHg}$. Fundal examination was completely obscured by a hypermature cataract and ocular B-scan ultrasound revealed discrete, mobile echogenic intravitreal particles (Fig. 1d). Examination of the left eye showed a low-grade anterior uveitis, which was successfully treated with topical drops.

He underwent an emergency right primary repair of inferior corneal laceration followed by lensectomy, surgical posterior capsulotomy and vitrectomy, which revealed extensive contamination of the vitreous with paint particles (Fig. 1e). The vitreous sample was sent for histopathology analysis (Fig. 2a-e). A few weeks later, a progressive inferior corneoscleral melt was observed, necessitating further surgeries, such as allogeneic lamellar sclero-corneal patch graft, amniotic membrane graft and ultimately a penetrating keratoplasty. At final follow-up, his corneal graft remained clear with a normal IOP and correcteddistance-visual-acuity of 6/36 (Fig. 1f).

Ocular tattooing has been used to treat disfiguring corneal scar, intractable diplopia and glare [2]. However, these invasive procedures can potentiate sight-threatening complications, especially, when performed by non-medically trained personnel [3]. Episcleral or conjunctival tattooing was first described in 2007 and, so far, there are several reports highlighting the significant complications associated with this type of procedure, including severe intraocular inflammation, cataract, secondary glaucoma, orbital cellulitis, scleritis and globe perforation [3, 4]. These complications arise either from the direct injury of the injection or from the hypersensitivity reaction to the constituents or contaminants of the injected pigments [3]. In addition, patients may not readily disclose the entire history of selftattooing complicating the diagnosis and management of the injury [3], as highlighted in our case.

The easy accessibility to social media in the current generation may act as a double-edged sword. With the rapid proliferation of health information online, it is becoming a common culture where patients turn to the internet as their first source of information and guidance on self-care procedures [5]. Clinicians need to maintain a low index of suspicion for self-treatment when encountering unexplained injuries to enable timely recognition and intervention of the complications. Awareness of these sight-threatening complications needs to be raised amongst the health professionals, general public and regulatory bodies.

Funding DSJT is supported by the Fight for Sight/John Lee, Royal College of Ophthalmologists Primer Fellowship.

\section{Compliance with ethical standards}

Conflict of interest The authors declare that they have no conflict of interest.

Publisher's note: Springer Nature remains neutral with regard to jurisdictional claims in published maps and institutional affiliations.

\section{References}

1. Braverman PK. Body art: piercing, tattooing, and scarification. Adolesc Med Clin. 2006;17:505e519.

2. Islam N, Franks WA. Therapeutic corneal tattoo following peripheral iridotomy complication. Eye (Lond). 2006;20:389-90.

3. Jalil A, Ivanova T, Bonshek R, Patton N. Unique case of eyeball tattooing leading to ocular penetration and intraocular tattoo pigment deposition. Clin Exp Ophthalmol. 2015;43:594-6.

4. Cruz NF, Santos KS, Farah ML, Felberg S. Conjunctival tattoo with inadvertent globe penetration and associated complications. Cornea. 2017;36:625-7.

5. Basch $\mathrm{CH}$, Basch CE, Hillyer GC, Reeves R. YouTube videos related to skin cancer: a missed opportunity for cancer prevention and control. JMIR Cancer. 2015;1:e1. 